\title{
Coupled second order singular perturbations for phase transitions
}

\author{
CMU 06/09/11
}

Ana Cristina Barroso, Margarida Baía, Milena Chermisi, JM 


\section{Introduction}

- Let $\Omega \subset \mathbb{R}^{d}$ with Lipschitz boundary ( container ) and let $u$ denote the configuration of the two-fluid system. 


\section{Introduction}

- Let $\Omega \subset \mathbb{R}^{d}$ with Lipschitz boundary ( container ) and let $u$ denote the configuration of the two-fluid system.

- Let $W$ be a nonnegative bulk free energy with

$$
\{W=0\}=\{a, b\} .
$$




\section{Introduction}

Gibb's criteria for equilibria leads to the study of the problem

$(P)$ minimize $\int_{\Omega} W(u(x)) d x$ under the constraint

$$
\begin{aligned}
& \int_{\Omega} u(x) d x=m \\
& \text { where } m=(\theta a+(1-\theta) b) \mathcal{L}^{d}(\Omega), 0<\theta<1 .
\end{aligned}
$$




\section{Introduction}

- Naturally $(P)$ may admit infinitely many solutions. 


\section{Introduction}

- Naturally $(P)$ may admit infinitely many solutions.

- In order to select physically preferred solutions, following the ideas of the gradient theory of phase transitions (Van der Waals, 1893), the following model was introduced by Cahn and Hilliard (1958) which assigns to each configuration $u$ of the two-fluid system an energy of the form:

$$
E_{\varepsilon}(u)=\int_{\Omega} W(u(x))+\varepsilon^{2}|\nabla u(x)|^{2} d x,
$$

where $\varepsilon>0$ is a small parameter. 


\section{Introduction}

- The competing effects of the two integrals favor separation of phases (i.e. configurations where $u$ takes values close to $a$ and $b$ ), while penalizing inhomogeneities of $u$ and, consequently, the introduction of too many transition regions. 


\section{Introduction}

- The competing effects of the two integrals favor separation of phases (i.e. configurations where $u$ takes values close to $a$ and $b$ ), while penalizing inhomogeneities of $u$ and, consequently, the introduction of too many transition regions.

- Gurtin (1983) conjectured that solutions of $\left(P_{\varepsilon}\right)$ minimize $E_{\varepsilon}(u)$ under the constraint $\int_{\Omega} u(x) d x=m$ converge to minimizers of $(P)$ having minimal interfacial energy. 


\section{Introduction}

- Gurtin's conjecture was proved (using De Giorgi's notion of $\Gamma$-convergence and following the ideas of Modica and Mortola) ) by Carr, Gurtin and Slemrod (1984) in the scalar case $d=1$ and independently by Modica and Sternberg (1987 - 1988) in the higher dimensional case $d \geq 2$. 


\section{Introduction}

- Gurtin's conjecture was proved (using De Giorgi's notion of $\Gamma$-convergence and following the ideas of Modica and Mortola) ) by Carr, Gurtin and Slemrod (1984) in the scalar case $d=1$ and independently by Modica and Sternberg (1987 - 1988) in the higher dimensional case $d \geq 2$.

- The vectorial case $\left(u: \Omega \subset \mathbb{R}^{d} \rightarrow \mathbb{R}^{N}, d, N \geq 2\right)$ was considered by Fonseca and Tartar (1989), Sternberg (1991) and Barroso and Fonseca (1994). 


\section{Introduction}

Further generalizations (not exhaustive):

- case where $W$ has more than two wells (Baldo, Sternberg, Ambrosio); 


\section{Introduction}

Further generalizations (not exhaustive):

- case where $W$ has more than two wells (Baldo, Sternberg, Ambrosio);

- coupled problem (Bouchitte', Owen and Sternberg, Fonseca and Popovici). 


\section{Introduction}

- The characterization of the $\Gamma$-limit for functionals involving second order terms ( in the context of elastic solid-solid phase transitions) is due to Conti, Fonseca, Leoni for an energy of the form:

$$
\int_{\Omega} W(\nabla u)+\epsilon^{2}\left|\nabla^{2} u\right|^{2} d x .
$$




\section{Introduction}

- The characterization of the $\Gamma$-limit for functionals involving second order terms (in the context of elastic solid-solid phase transitions) is due to Conti, Fonseca, Leoni for an energy of the form:

$$
\int_{\Omega} W(\nabla u)+\epsilon^{2}\left|\nabla^{2} u\right|^{2} d x .
$$

- We refer also to Chermisi, Dal Maso, Fonseca, Leoni, for a model on pattern formation based on Ginzburg-Landau energy

$$
\int_{\Omega} W(u)-q \epsilon^{2}|\nabla u|^{2}+\epsilon^{4}\left|\nabla^{2} u\right|^{2} d x, \quad q>0
$$




\section{Statement of the problem}

In this work (two-wells, coupled, vectorial, dependence on second gradient ) we consider the sequence of energy functionals

$$
\frac{1}{\varepsilon} \int_{\Omega} f\left(x, u(x), \varepsilon \nabla u(x), \varepsilon^{2} \nabla^{2} u(x)\right) d x
$$




\section{Statement of the problem}

In this work (two-wells, coupled, vectorial, dependence on second gradient ) we consider the sequence of energy functionals

$$
\frac{1}{\varepsilon} \int_{\Omega} f\left(x, u(x), \varepsilon \nabla u(x), \varepsilon^{2} \nabla^{2} u(x)\right) d x
$$

where $u \in W^{2,2}\left(\Omega ; \mathbb{R}^{N}\right)$ is a vector-valued function which describes the state of the mixture of $N$ fluids $(N \in \mathbb{N}, N \geq 2), \Omega$ is an open bounded subset of $\mathbb{R}^{d}$ $(d \in \mathbb{N}, d \geq 2)$ with Lipschitz boundary which represents the container and $\varepsilon>0$ is a small parameter. 


\section{Statement of the problem}

We assume that each scalar component of $u$, which identifies the density of an ingredient of the mixture is nonnegative, that is,

$$
u \in \mathbb{R}_{+}^{N}:=[0,+\infty)^{N} .
$$




\section{Statement of the problem}

We assume that each scalar component of $u$, which identifies the density of an ingredient of the mixture is nonnegative, that is,

$$
u \in \mathbb{R}_{+}^{N}:=[0,+\infty)^{N} .
$$

Therefore, the bulk energy density $f(x, u, \xi, \Lambda)$ is defined on $\Omega \times \mathbb{R}_{+}^{N} \times \mathbb{R}^{N \times d} \times \mathcal{T}^{N \times N \times d}$, where we denote by $\mathrm{Sym}^{N}$ the space of symmetric $N \times N$ matrices and by $\mathcal{T}^{N \times N \times d}$ the space of tensors $\Lambda=\left(\Lambda^{1}, \ldots, \Lambda^{d}\right), \Lambda^{i} \in \operatorname{Sym}^{N}, i=1, \ldots, d$. 


\section{Statement of the problem}

\section{We assume that}




\title{
Statement of the problem
}

\section{We assume that}

$$
[(\mathrm{H} 1)] f: \Omega \times \mathbb{R}_{+}^{N} \times \mathbb{R}^{N \times d} \times \mathcal{T}^{N \times N \times d} \rightarrow[0,+\infty) \text { is }
$$

\author{
continuous;
}




\section{Statement of the problem}

\section{We assume that}

$$
[(\mathrm{H} 1)] f: \Omega \times \mathbb{R}_{+}^{N} \times \mathbb{R}^{N \times d} \times \mathcal{T}^{N \times N \times d} \rightarrow[0,+\infty) \text { is }
$$
continuous;

$[(\mathrm{H} 2)] f(x, u, \mathrm{O}, \mathrm{O})=0$ if and only if $u \in\{\alpha, \beta\} \subset \mathbb{R}_{+}^{N}, \alpha \neq \beta$; 


\section{Statement of the problem}

$[(\mathrm{H} 3)]$ there exists a continuous function

$g: \bar{\Omega} \times \mathbb{R}_{+}^{N} \rightarrow[0,+\infty)$ such that

$\frac{1}{C_{0}}\left(g(x, u)+|\xi|^{2}+|\Lambda|^{2}\right) \leq f(x, u, \xi, \Lambda) \leq C_{0}\left(g(x, u)+|\xi|^{2}+|\Lambda|^{2}\right)$

for all $(x, u, \xi, \Lambda) \in \Omega \times \mathbb{R}_{+}^{N} \times \mathbb{R}^{N \times d} \times \mathcal{T}^{N \times N \times d}$, where $g$ satisfies

$$
\frac{1}{C_{0}}|u|^{q}-C_{0} \leq g(x, u) \leq C_{0}\left(1+|u|^{q}\right)
$$

for some $q \geq 2$, some $C_{0}>0$, and for all $(x, u) \in \bar{\Omega} \times \mathbb{R}_{+}^{N}$; 


\section{Statement of the problem}

$[(\mathrm{H} 4)]$ for every $x_{0} \in \Omega$ and every $\tau>0$ there exists $\delta>0$ such that $\left|x-x_{0}\right|<\delta$ implies

$$
\left|f(x, u, \xi, \Lambda)-f\left(x_{0}, u, \xi, \Lambda\right)\right| \leq \tau f(x, u, \xi, \Lambda)
$$

for all $(u, \xi, \Lambda) \in \mathbb{R}_{+}^{N} \times \mathbb{R}^{N \times d} \times \mathcal{T}^{N \times N \times d}$; 


\section{Statement of the problem}

$[(\mathrm{H} 4)]$ for every $x_{0} \in \Omega$ and every $\tau>0$ there exists $\delta>0$ such that $\left|x-x_{0}\right|<\delta$ implies

$$
\left|f(x, u, \xi, \Lambda)-f\left(x_{0}, u, \xi, \Lambda\right)\right| \leq \tau f(x, u, \xi, \Lambda)
$$

for all $(u, \xi, \Lambda) \in \mathbb{R}_{+}^{N} \times \mathbb{R}^{N \times d} \times \mathcal{T}^{N \times N \times d}$;

[(H5)] for every $M>0$ there exists $C_{M}>0$ such that for every $u_{1}, u_{2} \in \mathbb{R}_{+}^{N}$ with $\left|u_{1}\right|,\left|u_{2}\right| \leq M$, and every $(x, \xi, \Lambda) \in \Omega \times \mathbb{R}^{N \times d} \times \mathcal{T}^{N \times N \times d}$,

$$
\left|f\left(x, u_{1}, \xi, \Lambda\right)-f\left(x, u_{2}, \xi, \Lambda\right)\right| \leq C_{M}\left|u_{1}-u_{2}\right|\left(1+|\xi|^{2}+|\Lambda|^{2}\right) ;
$$




\section{Statement of the problem}

$[(\mathrm{H} 6)]$ there exist $\delta_{0}>0$ and $C>0$ such that

$$
f(x, u, \mathrm{O}, \mathrm{O}) \leq C|u-\alpha|^{2}
$$

whenever $|u-\alpha|<\delta_{0}$ and $x \in \Omega$, and

$$
f(x, u, \mathrm{O}, \mathrm{O}) \leq C|u-\beta|^{2}
$$

whenever $|u-\beta| \leq \delta_{0}$ and $x \in \Omega$. 


\section{Statement of the problem}

We also assume that the total amount of bulk material is preserved, i.e.,

$$
\int_{\Omega} u(x) d x=V
$$

for some $V=\left(V^{1}, \ldots, V^{N}\right) \in \mathbb{R}_{+}^{N}$ satisfying

$$
|\Omega| \alpha^{i} \leq V^{i} \leq|\Omega| \beta^{i}, \quad \text { for every } i=1, \ldots, N,
$$

where $\alpha^{i}$ and $\beta^{i}$ are the $i$-th components of $\alpha$ and $\beta$, respectively, and $|\Omega|$ denotes the Lebesgue measure of $\Omega$. We write

$$
\mathcal{V}:=\{u:(0.1),(0.2) \text { hold }\} .
$$




\section{Statement of the problem}

The aim of this paper is to study the asymptotic behavior as, $\varepsilon \rightarrow 0^{+}$, of the sequence of functionals

$$
\begin{aligned}
E_{\varepsilon}(u ; \Omega):= & \frac{1}{\varepsilon} \int_{\Omega} f\left(x, u(x), \varepsilon \nabla u(x), \varepsilon^{2} \nabla^{2} u(x)\right) d x, \\
& \text { for } u \in W^{2,2}\left(\Omega ; \mathbb{R}_{+}^{N}\right),
\end{aligned}
$$

subject to the constraints (0.1) and (0.2). 


\section{BV functions}

We briefly recall some basic facts about functions of bounded variation. 


\section{BV functions}

We briefly recall some basic facts about functions of bounded variation.

A function $u \in L^{1}\left(\Omega ; \mathbb{R}^{N}\right)$ is a function of bounded variation, briefly $u \in B V\left(\Omega ; \mathbb{R}^{N}\right)$, if its distributional derivative $D u$ is a bounded ${ }^{N \times d}$-valued Radon measure. This means that for all $\psi \in C_{c}^{1}(\Omega)$ the integration-by-parts formula

$$
\int_{\Omega} \frac{\partial \psi}{\partial x_{j}} u^{i} d x=\int_{\Omega} \psi D u_{j}^{i}, \quad i=1, \ldots, N, j=1, \ldots, d,
$$

holds. 


\section{BV functions}

Clearly, we have that $u \in W^{1,1}\left(\Omega ; \mathbb{R}^{N}\right)$ when $D u \in L^{1}\left(\Omega ; \mathbb{R}^{N}\right)$ and the measures $D u_{j}^{i}$ are absolutely continuous with respect to the Lebesgue measure. 


\section{BV functions}

Clearly, we have that $u \in W^{1,1}\left(\Omega ; \mathbb{R}^{N}\right)$ when $D u \in L^{1}\left(\Omega ; \mathbb{R}^{N}\right)$ and the measures $D u_{j}^{i}$ are absolutely continuous with respect to the Lebesgue measure. Let $\Omega_{u}$ be the set of points where the approximate limit of $u$ exists, i.e., $x \in \Omega$ such that there exist $z \in \mathbb{R}^{N}$ with

$$
\lim _{\varepsilon \rightarrow 0} f_{Q(x, \varepsilon)}|u(y)-z| d y=0 .
$$

If $x \in \Omega_{u}$ and $z=u(x)$ we say that $u$ is approximately continuous at $x$ (or that $x$ is a Lebesgue point of $u$ ). The function $u$ is approximately continuous $\mathcal{L}^{d}$-a.e. $x \in \Omega_{u}$ and

$$
\mathcal{L}^{d}\left(\Omega \backslash \Omega_{u}\right)=0 .
$$




\section{BV functions}

We have the following Lebesgue-Radon-Nikodým decomposition

$$
D u=\nabla u \mathcal{L}^{d}\left\llcorner\Omega+D^{s} u\right.
$$

where $\nabla u \in L^{1}\left(\Omega ; \mathbb{R}^{N \times d}\right)$ is the density of the absolutely continuous part of $D u$ and $D^{s} u$ is the singular part of $D u$ with respect to $\mathcal{L}^{d}$. 


\section{BV functions}

Let $S_{u}$ be the jump set of this function, i.e., the set of points $x \in \Omega \backslash \Omega_{u}$ for which there exists $a, b \in \mathbb{R}^{N}$ and a unit vector $\nu \in S^{d-1}$, normal to $S_{u}$ at $x$, such that $a \neq b$ and

$$
\lim _{\varepsilon \rightarrow 0^{+}} \frac{1}{\varepsilon^{d}} \int_{\left\{y \in Q_{\nu}(x, \varepsilon):(y-x) \cdot \nu>0\right\}}|u(y)-a| d y=0
$$

and

$$
\lim _{\varepsilon \rightarrow 0^{+}} \frac{1}{\varepsilon^{d}} \int_{\left\{y \in Q_{\nu}(x, \varepsilon):(y-x) \cdot \nu<0\right\}}|u(y)-b| d y=0 .
$$

The triple $(a, b, \nu)$ uniquely determined by (0.6) and (0.7) up to permutation of $(a, b)$, and a change of sign of $\nu$ and is denoted by $\left(u^{+}(x), u^{-}(x), \nu_{u}(x)\right)$. 


\section{BV functions}

We report the following Structure Theorem for $B V$-functions.

We need first some terminology: $\mathrm{A} \mathcal{H}^{d-1}$-measurable set $E \subset \mathbb{R}^{d}$ is said to be a countably $\mathcal{H}^{d-1}$-rectifiable set if it can be covered $\mathcal{H}^{d-1}$ almost everywhere by a countable family of $(d-1)$-dimensional surfaces of class $C^{1}$. 


\section{BV functions}

We report the following Structure Theorem for $B V$-functions.

We need first some terminology: $\mathrm{A} \mathcal{H}^{d-1}$-measurable set $E \subset \mathbb{R}^{d}$ is said to be a countably $\mathcal{H}^{d-1}$-rectifiable set if it can be covered $\mathcal{H}^{d-1}$ almost everywhere by a countable family of $(d-1)$-dimensional surfaces of class $C^{1}$.

Theorem 0.2 (Structure Theorem for $B V$-functions ). If $\Omega \subset \mathbb{R}^{d}$ is open and $u \in B V\left(\Omega ; \mathbb{R}^{N}\right)$, then $S_{u}$ is a countably $\mathcal{H}^{d-1}$-rectifiable set oriented by $\nu_{u}$, and $D^{s} u$ can be decomposed as $D^{c} u+D^{j} u$, where $\left|D^{c} u\right|(E)=0$ for every Borel set $E$ with $\mathcal{H}^{d-1}(E)<\infty$, and

$$
D^{j} u=\left(u^{+}-u^{-}\right) \otimes \nu_{u} \mathcal{H}^{d-1}\left\llcorner S_{u} .\right.
$$




\section{Limiting energy}

To describe the limiting energy and state the main theorem, we need to introduce some notation.

Given $\nu \in \mathbb{S}^{d-1}:=\left\{x \in \mathbb{R}^{d}:\|x\|=1\right\}$ let $\left\{\nu_{1}, \ldots, \nu_{d-1}, \nu\right\}$ be an orthonormal basis of $\mathbb{R}^{d}$,

Let $S_{\nu}$ be the strip:

$$
S_{\nu}:=\left\{x \in \mathbb{R}^{d}:|y \cdot \nu|<\frac{1}{2}\right\}
$$

and let $Q_{\nu}$ denote an open unit cube centered at the origin with two of its faces normal to $\nu$. 


\section{Limiting energy}

We define the class of admissible density functions by

$$
\begin{aligned}
& \mathcal{A}(\nu):=\left\{w \in W_{\mathrm{loc}}^{2,2}\left(S_{\nu} ; \mathbb{R}_{+}^{N}\right): w(y)=\alpha \text { if } y \cdot \nu=-\frac{1}{2},\right. \\
& w(y)=\beta \text { if } y \cdot \nu=\frac{1}{2} \\
& w(y)=w\left(y+k \nu_{i}\right), \\
& \text { for all } \left.y \in S_{\nu}, \quad i=1, \ldots, d-1, \text { and } k \in \mathbb{Z}\right\} .
\end{aligned}
$$




\section{Limiting energy}

We also introduce the surface energy density

$\sigma: \Omega \times \mathbb{S}^{d-1} \rightarrow[0,+\infty)$ defined by

$$
\begin{gathered}
\sigma(x, \nu):=\inf \left\{\quad \int_{Q_{\nu}} \frac{1}{t} f\left(x, w(y), t \nabla w(y), t^{2} \nabla^{2} w(y)\right) d y\right. \\
t>0, w \in \mathcal{A}(\nu)\} .
\end{gathered}
$$




\section{Limiting energy}

The limiting energy $F: L^{1}\left(\Omega ; \mathbb{R}_{+}^{N}\right) \rightarrow[0,+\infty]$ is given by

$F(u ; \Omega):=\left\{\begin{array}{l}\int_{S_{u}} \sigma\left(x, \nu_{u}(x)\right) d \mathcal{H}^{d-1}(x) \text { if } u \in B V(\Omega ;\{\alpha, \beta\}) \cap \mathcal{V} \\ +\infty \text { otherwise }\end{array}\right.$

where $S_{u}$ is the set of approximate jump points of $u$ and $\nu_{u}$ is the generalized unit inner normal to $S_{u}$ 


\section{Main result}

Theorem 0.3. Let $\Omega$ be a bounded open subset of $\mathbb{R}^{d}$ with Lipschitz boundary. Assume further that (H1)-(H6) hold. Then, for every $\varepsilon_{n} \rightarrow 0^{+}$ as $n \rightarrow+\infty$, the sequence of functionals

$F_{\varepsilon_{n}}: L^{1}\left(\Omega ; \mathbb{R}_{+}^{N}\right) \rightarrow[0,+\infty]$ defined by

$$
F_{\varepsilon_{n}}(u ; \Omega):= \begin{cases}E_{\varepsilon_{n}}(u ; \Omega) & \text { if } u \in W^{2,2}\left(\Omega ; \mathbb{R}_{+}^{N}\right) \cap \mathcal{V} \\ +\infty & \text { otherwise }\end{cases}
$$

$\Gamma$-converges, with respect to the $L^{1}\left(\Omega ; \mathbb{R}_{+}^{N}\right)$-convergence, to the limiting functional $F: L^{1}\left(\Omega ; \mathbb{R}_{+}^{N}\right) \rightarrow[0,+\infty]$ defined in $(0.10)$. 


\section{Remark}

Hypotheses (H5) and (H6) are needed in order to comply with the volume constraint. 


\section{$\Gamma$-convergence}

Taking into account the definition of $\Gamma$-convergence, in order to prove Theorem 0.3 it suffices to show:

(Lower bound) For every $u \in L^{1}\left(\Omega ; \mathbb{R}_{+}^{N}\right)$, for every sequence $\epsilon_{n} \rightarrow 0^{+}$and for every sequence $u_{n} \rightarrow u$ in $L^{1}\left(\Omega ; \mathbb{R}_{+}^{N}\right)$,

$$
F(u) \leq \liminf _{n \rightarrow \infty} F_{\epsilon_{n}}\left(u_{n}\right),
$$




\section{$\Gamma$-convergence}

(Upper bound) For every $\eta>0$, every $u \in L^{1}\left(\Omega ; \mathbb{R}_{+}^{N}\right)$, and every sequence $\epsilon_{n} \rightarrow 0^{+}$, there exists $\left\{u_{n}\right\} \subset L^{1}\left(\Omega ; \mathbb{R}_{+}^{N}\right)$ such that $u_{n} \rightarrow u$ in $L^{1}\left(\Omega ; \mathbb{R}_{+}^{N}\right)$ and

$$
\limsup _{n \rightarrow \infty} F_{\epsilon_{n}}\left(u_{n}\right) \leq F(u)+\eta \text {. }
$$




\section{Sketch of proofs}

The first step is to show that for every sequence

$u_{n} \in W^{2,2}\left(\Omega ; \mathbb{R}_{+}^{N}\right) \cap \mathcal{V}$ such that $u_{n} \rightarrow u$ in $L^{1}\left(\Omega ; \mathbb{R}_{+}^{N}\right)$ and for every $\varepsilon_{n} \rightarrow 0^{+}$we have that

$$
\liminf _{n \rightarrow \infty} \int_{\Omega} \frac{1}{\varepsilon_{n}} f\left(x, u_{n}(x), \varepsilon_{n} \nabla u_{n}(x), \varepsilon_{n}^{2} \nabla^{2} u_{n}(x)\right) d x=+\infty,
$$

unless $u \in B V(\Omega ;\{\alpha, \beta\})$. 


\section{Sketch of proofs}

The first step is to show that for every sequence

$u_{n} \in W^{2,2}\left(\Omega ; \mathbb{R}_{+}^{N}\right) \cap \mathcal{V}$ such that $u_{n} \rightarrow u$ in $L^{1}\left(\Omega ; \mathbb{R}_{+}^{N}\right)$ and for every $\varepsilon_{n} \rightarrow 0^{+}$we have that

$$
\liminf _{n \rightarrow \infty} \int_{\Omega} \frac{1}{\varepsilon_{n}} f\left(x, u_{n}(x), \varepsilon_{n} \nabla u_{n}(x), \varepsilon_{n}^{2} \nabla^{2} u_{n}(x)\right) d x=+\infty,
$$

unless $u \in B V(\Omega ;\{\alpha, \beta\})$. The proof (by contradiction) relies on hypotheses $\left(H_{2}\right)$ and $\left(H_{3}\right)$ and follows the ideas in Fonseca-Tartar. 


\section{Sketch of proofs}

Just an outline of the proof in the case we suppose $u=\beta \chi_{E}+\alpha\left(1-\chi_{E}\right)$ but $u \notin B V\left(\Omega ; \mathbb{R}_{+}^{N}\right)$ (i.e. $\left.\operatorname{Per}_{\Omega} E=+\infty\right)$. Suppose that there exist $\varepsilon_{n} \rightarrow 0^{+}$and $u_{n} \rightarrow u$ in $L^{1}\left(\Omega ; \mathbb{R}_{+}^{N}\right)$ such that

$$
\sup _{n} \int_{\Omega} \frac{1}{\varepsilon_{n}} f\left(x, u_{n}(x), \varepsilon_{n} \nabla u_{n}(x), \varepsilon_{n}^{2} \nabla^{2} u_{n}(x)\right) d x \leq C .
$$




\section{Sketch of proofs}

Just an outline of the proof in the case we suppose $u=\beta \chi_{E}+\alpha\left(1-\chi_{E}\right)$ but $u \notin B V\left(\Omega ; \mathbb{R}_{+}^{N}\right)$ (i.e. $\left.\operatorname{Per}_{\Omega} E=+\infty\right)$. Suppose that there exist $\varepsilon_{n} \rightarrow 0^{+}$and $u_{n} \rightarrow u$ in $L^{1}\left(\Omega ; \mathbb{R}_{+}^{N}\right)$ such that

$$
\sup _{n} \int_{\Omega} \frac{1}{\varepsilon_{n}} f\left(x, u_{n}(x), \varepsilon_{n} \nabla u_{n}(x), \varepsilon_{n}^{2} \nabla^{2} u_{n}(x)\right) d x \leq C .
$$

Defining $\bar{g}(u):=\min _{x \in \bar{\Omega}} g(x, u)$ where $g$ is the function in $\left(H_{3}\right)$ and

$\Phi(v):=\inf \left\{\int_{-1}^{1} \sqrt{\min \{\bar{g}(\psi(s)), M}\right\}\left|\psi^{\prime}(s)\right| d s$,

$\psi$ continuous and piecewise $\left.C^{1} \psi(-1)=\alpha, \psi(1)=v\right\}$, 


\section{Sketch of proofs}

we have that $\Phi$ is Lipschitz continuous and $\Phi \circ u=\chi_{E} \Phi(\beta)$ satisfies $|D(\Phi \circ u)|(\Omega)<+\infty$. Hence $\operatorname{Per}_{\Omega}(E)<\infty$, a contradiction. 


\section{Sketch of proofs}

Given the target function:

$$
u_{0}(x):= \begin{cases}\beta & \text { if } x \cdot \nu>0, \\ \alpha & \text { if } x \cdot \nu<0,\end{cases}
$$




\section{Sketch of proofs}

Given the target function:

$$
u_{0}(x):= \begin{cases}\beta & \text { if } x \cdot \nu>0, \\ \alpha & \text { if } x \cdot \nu<0,\end{cases}
$$

Using a slicing argument ( similarly to Chermisi-Dal MasoFonseca - Leoni) we derive a technical lemma that allows us to replace a sequence $\left\{v_{k}\right\}$ converging to $u_{0}$ in $L^{1}\left(\Omega ; \mathbb{R}_{+}^{N}\right)$ by a sequence $\left\{w_{k}\right\} \subset \mathcal{A}(\nu)$ still converging to $u_{0}$ in $L^{1}\left(\Omega ; \mathbb{R}_{+}^{N}\right)$, without increasing the total energy. 


\section{Sketch of proofs}

This sequence is obtained by an appropriate convex combination between $v_{k}$ and the convolution of $u_{0}$ ( on the cube $Q_{\nu}$ ) and then extended by periodicity to the strip $S_{\nu}$.

This technical result is used in both the lower and upper bound inequalities. 


\section{Sketch of proofs: lower bound}

Since

$$
f_{n}:=\int_{\Omega} \frac{1}{\varepsilon_{n}} f\left(x, u_{n}(x), \varepsilon_{n} \nabla u_{n}(x), \varepsilon_{n}^{2} \nabla^{2} u_{n}(x)\right) d x
$$

forms a sequence of nonnegative functions bounded in $L^{1}$ norm, (up to a subsequence) converges weakly* in the sense of measures to some nonnegative bounded Radon measure $\zeta$. 


\section{Sketch of proofs: lower bound}

Since

$$
f_{n}:=\int_{\Omega} \frac{1}{\varepsilon_{n}} f\left(x, u_{n}(x), \varepsilon_{n} \nabla u_{n}(x), \varepsilon_{n}^{2} \nabla^{2} u_{n}(x)\right) d x
$$

forms a sequence of nonnegative functions bounded in $L^{1}$ norm, (up to a subsequence) converges weakly* in the sense of measures to some nonnegative bounded Radon measure $\zeta$.

We just need to show that

$$
\zeta^{a}(x) \geq \sigma\left(x, \nu_{u}(x)\right), \text { for } \mathcal{H}^{d-1} \text { a.e. } x \in \Omega \cap S_{u},
$$

where $\zeta^{a}$ denotes the absolutely continuous part of $\zeta$ w.r.to the measure $\mathcal{H}^{d-1}\lfloor\partial E$. 


\section{Sketch of proofs: lower bound}

The result follows from Lebesgue Besicovitch derivation theorem since, choosing $r_{k} \rightarrow 0^{+}$s.t. $\zeta\left(\partial\left(x+r_{k} Q_{\nu}\right)\right)=0$, we have that

$$
\begin{aligned}
\zeta^{a}(x)= & \lim _{r \rightarrow 0^{+}} \frac{\zeta\left(Q_{\nu}(x ; r)\right)}{r^{d-1}} \\
& =\lim _{k \rightarrow \infty} \frac{1}{r_{k}^{d-1}} \lim _{n \rightarrow \infty} E_{\varepsilon_{n}}\left(u_{n} ; Q_{\nu}\left(x ; r_{k}\right)\right) .
\end{aligned}
$$




\section{Sketch of proofs: lower bound}

The result follows from Lebesgue Besicovitch derivation theorem since, choosing $r_{k} \rightarrow 0^{+}$s.t. $\zeta\left(\partial\left(x+r_{k} Q_{\nu}\right)\right)=0$, we have that

$$
\begin{aligned}
\zeta^{a}(x)= & \lim _{r \rightarrow 0^{+}} \frac{\zeta\left(Q_{\nu}(x ; r)\right)}{r^{d-1}} \\
& =\lim _{k \rightarrow \infty} \frac{1}{r_{k}^{d-1}} \lim _{n \rightarrow \infty} E_{\varepsilon_{n}}\left(u_{n} ; Q_{\nu}\left(x ; r_{k}\right)\right) .
\end{aligned}
$$

Change variables ( to the unit cube), diagonalization provedure and then apply the technical lemma in order to get a sequence in $\mathcal{A}(\nu)$. 


\section{Sketch of proofs: upper bound}

- Recall: (Upper bound) For every $\eta>0$, every $u \in L^{1}\left(\Omega ; \mathbb{R}_{+}^{N}\right)$, and every sequence $\epsilon_{n} \rightarrow 0^{+}$, there exists $\left\{u_{n}\right\} \subset L^{1}\left(\Omega ; \mathbb{R}_{+}^{N}\right)$ such that $u_{n} \rightarrow u$ in $L^{1}\left(\Omega ; \mathbb{R}_{+}^{N}\right)$ and

$$
\limsup _{n \rightarrow \infty} F_{\epsilon_{n}}\left(u_{n}\right) \leq F(u)+\eta
$$




\section{Sketch of proofs: upper bound}

- Recall: (Upper bound) For every $\eta>0$, every $u \in L^{1}\left(\Omega ; \mathbb{R}_{+}^{N}\right)$, and every sequence $\epsilon_{n} \rightarrow 0^{+}$, there exists $\left\{u_{n}\right\} \subset L^{1}\left(\Omega ; \mathbb{R}_{+}^{N}\right)$ such that $u_{n} \rightarrow u$ in $L^{1}\left(\Omega ; \mathbb{R}_{+}^{N}\right)$ and

$$
\limsup _{n \rightarrow \infty} F_{\epsilon_{n}}\left(u_{n}\right) \leq F(u)+\eta .
$$

- By blow-up techniques ( Fonseca - Muller ) to reduce the problem to the case where $u$ is like the target function $u_{0}$. 


\section{Sketch of proofs: upper bound}

- Recall: (Upper bound) For every $\eta>0$, every $u \in L^{1}\left(\Omega ; \mathbb{R}_{+}^{N}\right)$, and every sequence $\epsilon_{n} \rightarrow 0^{+}$, there exists $\left\{u_{n}\right\} \subset L^{1}\left(\Omega ; \mathbb{R}_{+}^{N}\right)$ such that $u_{n} \rightarrow u$ in $L^{1}\left(\Omega ; \mathbb{R}_{+}^{N}\right)$ and

$$
\limsup _{n \rightarrow \infty} F_{\epsilon_{n}}\left(u_{n}\right) \leq F(u)+\eta .
$$

- By blow-up techniques ( Fonseca - Muller ) to reduce the problem to the case where $u$ is like the target function $u_{0}$. 


\section{Sketch of proofs: upper bound}

This is done step by step relying on the following property of $\sigma$ that results from $(H 4)$ : 


\section{Sketch of proofs: upper bound}

This is done step by step relying on the following property of $\sigma$ that results from $(H 4)$ :

For all $\left(x_{0}, \nu\right) \in \Omega \times \mathbb{S}^{d-1}$ and $\tau>0$ there exists $\delta>0$ such $\left|x-x_{0}\right|<\delta$ implies that

$$
\left|\sigma(x, \nu)-\sigma\left(x_{0}, \nu\right)\right| \leq \tau C\left(1+|\alpha|^{q}+|\beta|^{q}+|\alpha-\beta|^{2}\right) .
$$




\section{Sketch of proofs: upper bound}

- first for a cube assuming no explicit dependence on $x$, 


\section{Sketch of proofs: upper bound}

- first for a cube assuming no explicit dependence on $x$,

- then for a cube allowing for dependence on $x$, 


\section{Sketch of proofs: upper bound}

- first for a cube assuming no explicit dependence on $x$,

- then for a cube allowing for dependence on $x$,

- next for a planar interface ( using Whitney's Covering Theorem ), 


\section{Sketch of proofs: upper bound}

- first for a cube assuming no explicit dependence on $x$,

- then for a cube allowing for dependence on $x$,

- next for a planar interface ( using Whitney's Covering Theorem ),

- then to a polygonal interface and finally for arbitrary sets of finite perimeter (by standard approximation results of sets of finite perimeter by polyhedral sets). 


\section{Upper bound}

In each step we construct a sequence $v_{n, \eta}$ satisfying the upper bound inequality and then change it in order to comply with the volume constraint through:

$$
u_{n, \eta}:=v_{n, \eta}+b_{n, \eta}, \quad b_{n, \eta}:=\frac{1}{|\Omega|} \int_{\Omega} u_{0}-v_{n, \eta} d x,
$$

and rely on $\left(H_{5}\right)$ and $\left(H_{6}\right)$ so that the upper bound inequality still holds. 


\section{Bibliography}

A. C. Barroso and I. Fonseca, Anisotropic singular perturbations - the vectorial case, Proc. Roy. Soc. Edinburgh, A 124 (1990).

M. Chermisi, G. Dal Maso, I. Fonseca and G. Leoni, Singular perturbation models in phase transitions for second order materials, Indiana Univ. Math. J., to appear.

I. Fonseca and C. Popovivi, Coupled singular perturbations for phase transitions, Asymptotic Anal. 44 (2005).

I. Fonseca and L. Tartar, The gradient theory of phase transitions for systems with two potential wells, Proc. Roy. Soc. Edinburgh, A 111 (1989).

M. Gurtin, On phase transitions with bulk, interfacial and boundary energy, Arch. Rational Mech. Anal. 96 (1986). 